\title{
On-line Measurement System for Anti icing Agent Content of Jet Fuel Based on Near Infrared Spectroscopy
}

\author{
Fanqin Meng ${ }^{1}$, a, Xiaozhong Zhang ${ }^{1,}$ b \\ ${ }^{1}$ Department of aviation oil ,Air Force Logistics college, Xuzhou 221000, China. \\ amfq6828@163.com \\ bzxzcn@china.com
}

\begin{abstract}
keywords: jet fuel , anti icing additive, infrared absorption spectroscopy, on-line measurement Abstract: For reducing the freezing possibility of water in jet fuel, it is necessary to add a certain amount of anti icing additive in jet fuel before refueling a plane. The jet fuel is a mixture contain of all variety of hydrocarbons and additives, so the online measurement of anti-icing additive concentration in jet fuel is difficult. In this paper, a method for measuring the concentration of anti-icing additive in jet fuel using near infrared(NIR) spectroscopy is studied. Based on this methods, The composition of the measurement system is put forward, and the on-line sampling device and the temperature control circuit are designed. According to the experimental data, it is proved that the system can realize the on-line measurement of the anti icing agent content and meet the requirements of the anti icing agent content measurement in the aircraft refueling.
\end{abstract}

In order to reduce the freezing possibility of water in jet fuel, A certain amount of anti icing additive is need. The anti-icing additive (ethylene glycol methyl fan is its main components) is easily extracted by water, and therefore cannot be added in factory like other additives, so it must be added in airport as refueling. In order to realize online control of the anti icing agent adding process and jet fuel quality detection, the online measurement technology of anti-icing additive concentration in jet fuel is need. But the jet fuel is a mixture contain of all variety of hydrocarbons and additives, its composition is complex and composition difference of product batches is remarkable, and only $0.1 \sim 0.15 \%$ the volume percent of Anti icing additive, so the additive concentration measurement is a typical problem of complex organic phase detection of trace substances. In this paper, a method for measuring the concentration of anti-icing additive in jet fuel using near infrared spectroscopy(NIR)is studied. Based on this methods, The composition of the measurement system is put forward, and the on-line sampling device and the temperature control circuit are designed.

\section{Measuring Principle}

Near infrared spectroscopy (NIR) technology is a comprehensive analysis technology based on near infrared spectroscopy and chemometrics analysis technology. It can be used for fast, non-destructive qualitative/ quantitative analysis of samples containing organic functional groups including $\mathrm{C}-\mathrm{H}$, $\mathrm{N}-\mathrm{H}$ and $\mathrm{O}-\mathrm{H}^{[1,2]}$. The measurement of the content of the anti ice agent in the jet fuel is based on the near infrared spectroscopy. Under laboratory conditions, we got the NIR absorption spectrums of jet fuel which containing 5 different concentrations of anti-icing additive. The spectrums show that there is a certain relationship between the concentration and the absorbance.

In order to find the quantitative relationship, mathematical models were established by multiple linear regression and principal component analysis method, a set of experiment data were used to test the models. The result show that the principal component regression model has the minimum error , of which the average relative error is $5.7 \%$, the average absolute error is $0.007 \%$. According to the study conclusions, the NIR method of measuring anti-icing addictive concentration in jet fuel can be proved to be feasible, Using this method can improve the measurement accuracy, short the measurement time. By the NIR method, an online measurement system of anti-icing addictive concentration can be developed. 


\section{Piping and Instrumentation Diagrams and Measuring Probe Structure}

The piping and instrumentation diagrams of the anti icing additive content measurement system is illustrated in Fig. 1. The sample enters the measurement device through the filter, the explosion-proof metering pump, and the three - way valve. The detection probe consists of a heat exchanger, a semiconductor refrigeration element and a flow cell, which is the key device for the measurement system. Because temperature has a great influence on the near infrared absorption spectrum ${ }^{[3]}$, the oil sampled has to be kept at a constant temperature . the measurement device ensures that the oil temperature is kept at $20{ }^{\circ} \mathrm{C}$ during the measurement process. The semiconductor refrigeration element is used here to achieve two-way temperature control function.

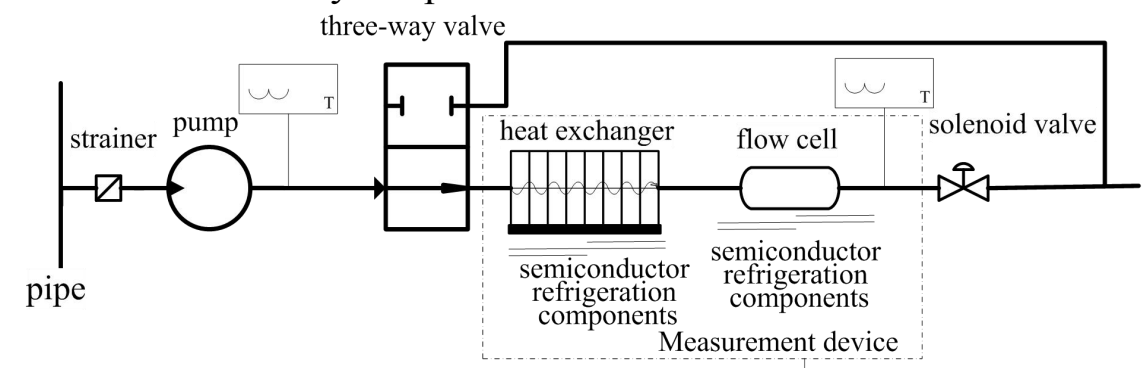

Fig.1 measurement system P\&ID

The measurement device structure is shown in Fig. 2. The heat exchange device of this detection probe is made of copper or aluminum alloy, and the sample capillary make heat exchange with the heat exchanger to adjust the temperature of the sample. The heating and cooling elements adopt semiconductor refrigeration element TEC1-12706. The maximum current is 4.7A when the working voltage is $12 \mathrm{~V}$, the thermal efficiency is $60 \%$, the maximum temperature difference is $65 \sim 69$ degrees, and the area is $40 \times 40 \times 3.8 \mathrm{~mm}$. When using semiconductor refrigeration element to control temperature, in order to achieve good refrigeration and heating effect, we must determine the driving voltage and current of semiconductor refrigeration element, and do thermal design of the hot end and cold end, reduce the heat resistance, prevent condensation in the cold side. This requires a complete isolation between the two sides of the cold and heat of the semiconductor refrigeration unit, small thermal resistance of the two contact surface, and a good adiabatic sealing for the external environment of the detection probe unit. One side of the semiconductor refrigeration element is heat exchange with the sample in the capillary tube by heating exchanger, heating or cooling the sample, the other side can use the plane heat pipe radiator to increase the heat dissipation effect, or the shell and the heat sink are bonded, and the entire shell is used as the heat sink.

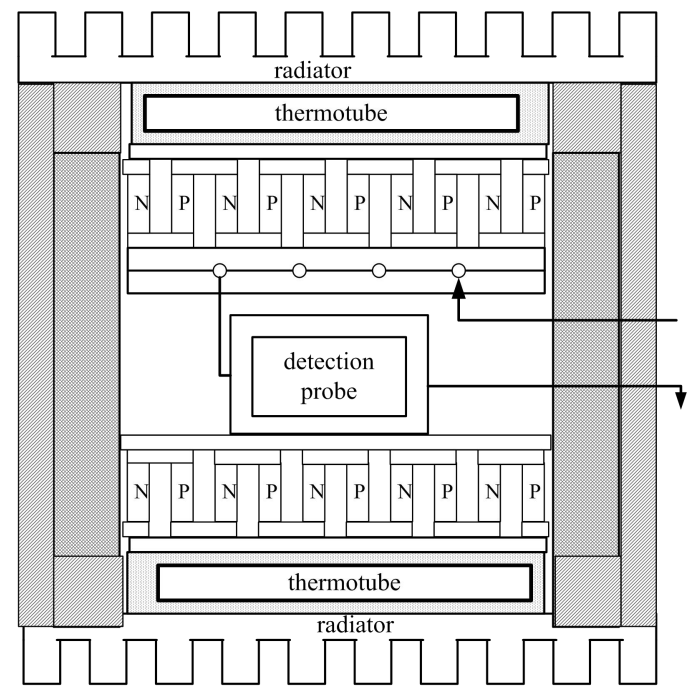

Fig. 2 measurement device structure

The natural convection heat transfer coefficient of air is $5 \sim 25\left(\mathrm{~W} / \mathrm{m}^{2} . \mathrm{k}\right)$. If the coefficient is 5 , the thermal power is $12 \mathrm{~W}$, and when the heat dissipation area is $0.25 \mathrm{~m}^{2}$, the temperature of the shell is 
raised to 9.6 degrees centigrade (relative to the ambient temperature). Assuming that the inner diameter of the heating pipe is $3 \mathrm{~mm}$, the pipe length of the $1 \mathrm{ml}$ sample is $142 \mathrm{~mm}$, and the TEC1-12706 working face is $40 \times 40 \mathrm{~mm}$, and the heating pipeline on the heating surface is designed as "M" shape. The temperature of the measuring probe is controlled by a semiconductor refrigeration unit in the same way of the temperature control of the sample, and the temperature is kept as 20 degrees Celsius. The temperature probe is embedded in the inlet, the outlet of the heating line and the measuring probe respectively. In actual work, the probe is preheated first, and the preheating time is not more than 2 minutes. The specific heat of steel is $450 \mathrm{~J} / \mathrm{kg}$. ${ }^{\circ} \mathrm{C}$. If the ambient temperature is $-25{ }^{\circ} \mathrm{C}$, the temperature rises to $45{ }^{\circ} \mathrm{C}$ when it is heated to $20{ }^{\circ} \mathrm{C}$. Assuming that the weight of the measuring probe is 250 grams, the heating power is $4.2 \mathrm{~W}$, less than $5 \mathrm{~W}$.

As shown in fig. 2, the detection probe is made of engineering plastics and is lined with insulating materials. The heating and measuring space is isolated from thermal insulation materials. Connection terminals, fiber optic joints and pipeline joints are set in the appropriate position.

\section{Measurement System Composition and Measurement Process}

On the basis of embedded computer, we extend the drive circuit of pump, valve and semiconductor refrigeration element, temperature measurement circuit and fiber spectrometer, constitute the online measurement system of jet fuel anti icing agent content, as shown in Fig.3.

The metering pump is controlled by an embedded system to determine the flow velocity and measurement interval of the sample. The temperature control device consists of a semiconductor cooling element and a heat exchange device. The temperature sensor measures the inlet and outlet temperature separately, and the embedded system control the current magnitude and direction of the semiconductor cooling element making it heating and cooling alternately ,so as to keep sample temperature as a constant.

The measurement time was set to $10 \mathrm{~s}$, and the volume of the probe was $1 \mathrm{ml}$. In Figure 2, the total capacity of the filter, pump and injection line (before the heating line) is set to $5 \mathrm{ml}$. The measurement temperature is determined to be 20 . The sample is divided into two steps. First, the sample that is left in the filter, sampling pump and the sampling line at the time of the last sampling is excluded. It is assumed that the flow rate of the injection pump is $2.5 \mathrm{ml} / \mathrm{s}(150 \mathrm{ml} / \mathrm{min})$, the time for the left sample to flow into the return pipeline through the three-way valve is $2 \mathrm{~S}$. The second step is to enter the sample. Control the three-way valve to drive the sample in the heating line into the measuring pool, and the sample of the measuring pool is discharged by the solenoid valve. At the same time, the heating line is filled with sample for the next measurement cycle. The flow rate of the injection pump is set to $0.5 \mathrm{ml} / \mathrm{s}(30 \mathrm{ml} / \mathrm{min})$, so the time for sample fill the probe chamber is $2 \mathrm{~S}$. After the sample was filled with the probe chamber and stabilized for $2 \mathrm{~S}$, the spectrometer began to measure. The measuring time of the spectrometer is set to 4 seconds. After the spectrometer saves the data, the embedded system begins to read the spectrometer data in ninth seconds. The timing sequence of the measurement is shown in Fig. 4. The temperature control device works all the time and stabilizes the sample at $20{ }^{\circ} \mathrm{C}$ within $10 \mathrm{~s}$.

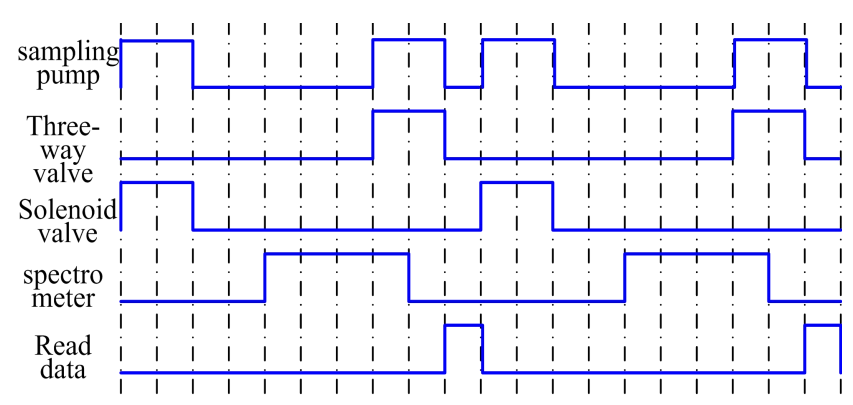

Fig. 3 measurement system composition

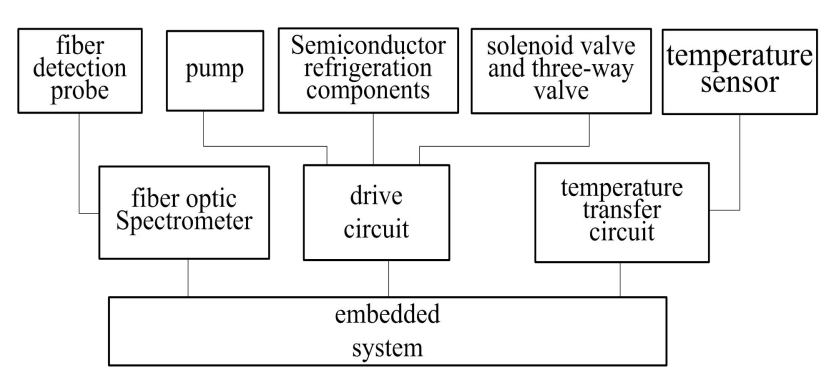

Fig. 4 sequence diagram

The specific heat of oil is assumed to be $2.14 \mathrm{kj} / \mathrm{kg} .{ }^{\circ} \mathrm{C}$, and the temperature range of the imported samples is from -15 to $30^{\circ} \mathrm{C}$. So in 10 seconds, the temperature rises by $35^{\circ} \mathrm{C}$, and the temperature 
decreases by $10^{\circ} \mathrm{C}$. If the volume of the measured sample is $1 \mathrm{ml}$ and the mass is $0.8 \mathrm{~g}$, the power of the heating is calculated to be $6 \mathrm{~W}$ and the power of the refrigeration is $1.8 \mathrm{~W}$. Considering the influence of ambient temperature, the power is increased by $50 \%$ respectively. That is, the heating power is set to $10 \mathrm{~W}$, and the cooling power is set to $3 \mathrm{~W}$. This power setting also adapts to the high heating power of the semiconductor components and the low efficiency of the refrigeration. When the heat end of TEC $1-12706$ is at $25^{\circ} \mathrm{C}$, the power can reach $50 \mathrm{~W}$, which can satisfy the requirement of temperature regulation.

\section{Experimental Results}

The test device for the anti ice agent has been experimentally verified. In the experiment, 5 samples of jet fuel with different antifreeze content were prepared. The contents of the samples were $0.05 \%$, $0.075 \%, 0.1 \%, 0.125 \%$ and $0.15 \%$ respectively. 45 pieces of near infrared spectrum data are collected, of which 30 are used as correction sets and 15 are used as verification sets. The experiment proved that the experimental device can quickly detect the content of anti ice agent. A near-infrared prediction model is set up with air as a reference object. The prediction results are shown in Table 1 . According to the experimental data, when the content of anti icing agent in jet fuel is below $0.15 \%$, the content of the anti icing agent can be quickly determined by near infrared spectroscopy, and the accuracy of the anti icing agent content can reach to $\pm 0.01 \%$.

Table.1 Forecast results

\begin{tabular}{|l|l|l|l|l|}
\hline No & Name of the sample & $\begin{array}{l}\text { reference } \\
\text { data(\%) }\end{array}$ & $\begin{array}{l}\text { Forecast } \\
\text { data }\end{array}$ & $\begin{array}{l}\text { Prediction } \\
\text { deviation (\%) }\end{array}$ \\
\hline 1 & 2_No.1_1712190016 & 0.05 & 0.059 & 0.009 \\
\hline 2 & 2_No.2_1712190017 & 0.05 & 0.055 & 0.005 \\
\hline 3 & 2_No.3_1712190018 & 0.05 & 0.061 & 0.011 \\
\hline 4 & 3_No.1_1712190025 & 0.075 & 0.083 & 0.008 \\
\hline 5 & 3_No.2_1712190026 & 0.075 & 0.079 & 0.004 \\
\hline 6 & 3_No.3_1712190027 & 0.075 & 0.077 & 0.002 \\
\hline 7 & 4_No.1_1712190034 & 0.1 & 0.089 & -0.011 \\
\hline 8 & 4_No.2_1712190035 & 0.1 & 0.089 & -0.011 \\
\hline 9 & 4_No.3_1712190036 & 0.1 & 0.089 & -0.011 \\
\hline 10 & 5_No.1_1712190043 & 0.125 & 0.107 & -0.018 \\
\hline 11 & 5_No.2_1712190044 & 0.125 & 0.11 & -0.015 \\
\hline 12 & 5_No.3_1712190045 & 0.125 & 0.11 & -0.015 \\
\hline 13 & 6_No.1_1712190052 & 0.15 & 0.145 & -0.005 \\
\hline 14 & 6_No.2_1712190053 & 0.15 & 0.149 & -0.001 \\
\hline 15 & 6_No.3_1712190054 & 0.15 & 0.145 & -0.005 \\
\hline SEV & 0.010 & & \\
\hline
\end{tabular}

\section{References}

[1]E.Dessipri,E.Minopoulou,G.D.Chryssikos, et al.Panayiotou Use of FT-NIR spectroscopy for on-line monitoring of formaldehyde-based resin synthe-sis, Eur Polym J[J].2003,39:1533-1540.

[2]Emilio Marengoa, Marco Bobbaa, Elisa Robotti. Hydroxyl and acid number prediction in polyester res-ins by near infrared spectroscopy and artificial neural networks. Anal Chim Acta [J]. 2004,511:313-322

[3]LaneS., MartinE.B., MorrisA.J., GowerP.. Application of exponentially weighted principal component analysis for the monitoring of a polymer film manufacturing process. Transactions of the Institute of Measurement and Control 25(1),17-35(2003) 